TITLE:

\title{
Numerical modelling of space and time variations of seismic activity before major earthquakes
}

\section{$\operatorname{AUTHOR}(\mathrm{S})$ :}

Mikumo, Takeshi; Miyatake, Takashi

\section{CITATION:}

Mikumo, Takeshi ... [et al]. Numerical modelling of space and time variations of seismic activity before major earthquakes. Geophysical Journal of the Royal Astronomical Society 1983, 74(2): 559-583

ISSUE DATE:

1983-05

URL:

http://hdl.handle.net/2433/193394

RIGHT:

(C) The Royal Astronomical Society 


\title{
Numerical modelling of space and time variations of seismic activity before major earthquakes
}

\author{
Takeshi Mikumo Disaster Prevention Research Institute, Kyoto University, \\ Uji, Kyoto 611, Japan
}

Takashi Miyatake Earthquake Research Institute, University of Tokyo, Tokyo 113, Japan

Received 1982 December 7; in original form 1982 July 13

Summary. The spatial and temporal variations of seismic activity before large earthquakes, including precursory swarm earthquakes, clustering, pre-seismic quiescence in the epicentral area, doughnut patterns and foreshocks, are numerically modelled by quasi-static and dynamical rupture processes on three-dimensional heterogeneous faults with various types of non-uniform strength distributions. The main physical parameters that could yield these precursory changes of seismicity patterns are the form of distributions of static and sliding frictional strengths and their spatial variations over the fault, and also the rate of increase of tectonic stress.

For weakly heterogeneous faults, a large-scale main shock rupturing the entire fault surface occurs without any precursory indications. If the fault has moderately to heavily non-uniform strengths on a small-scale, gradually increasing activity tends to occur over a long period prior to a main event, with no swarms or quiescence. For extremely heterogeneous faults including a number of small to moderate-size asperities with medium to high strengths, a typical sequence appears with swarms, quiescence, foreshocks and a main shock event. The temporal behaviour of these events seems to depend primarily on the form of distribution of the strengths. If the fault includes a large-size asperity with medium to high strengths in its central part, a doughnut pattern of seismicity repeatedly appears with high activity and quiescence in the surrounding and central asperity regions. If a belt-like barrier region with extremely high strengths lies in the central part of the fault, dividing the entire region into two sections, large-scale shocks take place successively in the adjacent unruptured regions at slightly different times, and a main shock event rupturing the entire fault occurs after a long period of quiescence.

These results from numerical simulations could provide possible explanations of observed seismicity patterns prior to major earthquakes.

\section{Introduction}

It is now widely recognized that there are several different types of spatial and temporal variations of seismic phenomena before large earthquakes. These precursory phenomena, 
including the change of seismicity patterns such as seismic gaps, precursory swarms, clustering, pre-seismic quiescence in the epicentral area, doughnut patterns, migrations, and foreshocks, as well as precursory slow fault slips, have often been observed to date prior to some major earthquakes. These observations seem extremely important for comprehensive understanding of the state of crustal stress and the physical process of earthquakes, and also for prediction purposes. A number of close investigations into these observations have been made in recent years, as well summarized in some of the recent literature (e.g. Ishida \& Kanamori 1980; Kanamori 1981).

Seismic space gaps have been identified in several plate boundaries along major oceanic trenches as zones for future possible sites for large earthquakes, where none has occurred for a long time (e.g. Fedotov 1965; Mogi 1968a,b; Sykes 1971; Kelleher, Sykes \& Oliver 1973; Utsu 1974; Kelleher \& Savino 1975; Ohtake, Matsumoto \& Latham 1977; McCann et al. 1979). Regional scale migrations of seismic activity, in which major earthquakes took place successively in adjacent regions, moving in one direction, have also been reported (e.g. Mogi $1968 \mathrm{c}$ ). Pre-seismic quiescence, which is identified as a temporal gap, has been noticed in many cases, indicating that seismicity in the epicentral area of an eventual large earthquake becomes very low before the main shock (e.g. Inouye 1965; Mogi 1968a; Ohtake et al. 1977). A doughnut pattern of seismicity sometimes appears with this quiescence, the surrounding regions becoming active (e.g. Mogi 1969; Yamashina \& Inouye 1979). Anomalously high activity or strong clustering of minor to moderate-size shocks has also been observed within or near the source area of impending large earthquakes (e.g. Mogi 1969; Sekiya 1977; Kelleher \& Savino 1975; Evison 1977a,b, c; Ohtake 1976; Ishida \& Kanamori 1978, 1980). Foreshock activity, on the other hand, precedes a large fraction of major earthquakes with greater magnitudes in certain regions (e.g. Mogi 1963b; Jones \& Molnar 1979), but some events even with comparable magnitudes and often with lesser magnitudes are not preceded by pronounced foreshock activity.

It has been pointed out (Evison 1977a,b,c) that one of the typical patterns of these observed sequences includes four episodes of seismicity changes; normal background seismicity, precursory swarms over a rather wide area, a long period of quiescence, and a main event and its aftershocks. A schematic space-time diagram of these patterns has been proposed by Mogi (1977) and slightly modified by Kanamori (1981), as illustrated in Fig. 1,

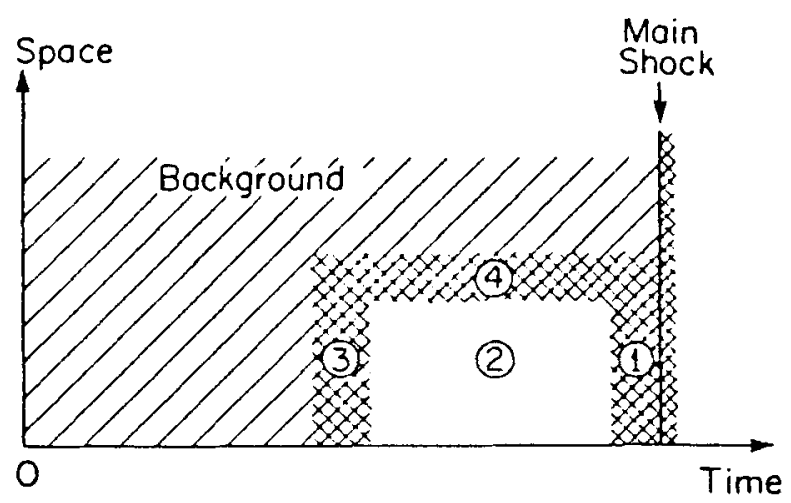

Figure 1. A schematic space diagram of seismicity patterns slightly modified from Mogi (1977) and Kanamori (1981). 1, foreshock activity; 2, pre-seismic quiescence in the epicentral area; 3 , precursory swarms or clustering; 4 , high activity in the surrounding regions, in contrast to the quiescence in the epicentral area, indicating a doughnut pattern. 
although actual seismic activity observed before major earthquakes is often more complicated, and their detailed features appear significantly different from place to place and from event to event. Actually, some large earthquakes are preceded by none or only part of the above described change of seismicity patterns. Besides these variations of seismicity, precursory slow fault slips have been recorded prior to some earthquakes (e.g. Kanamori \& Cipar 1974; Sacks et al. 1978; Fujii 1978). Although these premonitory slips are not always observed in major earthquakes, they might have some relation to a burst of foreshock activity (Jones \& Molnar 1979).

We have the standpoint that all these observations may be direct manifestations of the state of space- and time-dependent stress, with respect to the strength of rock materials, along and around the fault zone of these major earthquakes. The final goal of our studies is to postulate a possible physical model to account for the conditions under which the above precursory phenomena could arise. As a first step for the studies, we calcuiate, in this paper, numerical solutions for quasi-static and dynamical rupture process on heterogeneous faults over a long time range, taking into account some results from laboratory friction experiments on rock materials. Our attention will be focused here on how these heterogeneous distributions of frictional strengths, particularly of fault asperities, will affect the space and time characteristics of precursory phenomena before large earthquakes.

\section{Model}

We have shown in a previous paper (Mikumo \& Miyatake 1979), on the basis of a threedimensional, dynamic fault model (Mikumo \& Miyatake 1978), that heterogeneous faults with non-uniform distribution of frictional strengths and relaxation times, which are subjected to time-dependent shear stress, could account for the space and time characteristics of earthquake sequences including a main shock, aftershocks and the recurrence of major shocks in a long time range. These heterogeneous faults were introduced to include portions with strong and weak strengths, which are represented by the bi-modal Weibull distributions and with various scale lengths, indicating different degrees of contact of fault surfaces, such as asperities, fault geometries and inhomogeneous pore pressures etc. It has been shown (Mikumo \& Miyatake 1978) that these heterogeneous models could also well explain the initiation, spreading and stopping of dynamic ruptures, incoherent rupture propagation, nonuniform fault displacements and radiation of high-frequency seismic waves. The numerical experiments by Mikumo \& Miyatake (1979) have clearly indicated the following spatial and temporal features: major shocks may take place successively in adjacent regions on the fault so as to fill the space gaps, and sometimes with slow-speed migrations, and the recurrence of large earthquakes is often preceded by a complete time gap or very low activity with a small number of foreshocks. Kanamori (1981) proposed a simple asperity model to explain the natures of various seismicity patterns. Although this is a two-dimensional, static model with high strength asperities, he was also able to explain, to some extent, temporal variations of seismicity with swarms and quiescence. Jones \& Molnar (1979) also assumed an inhomogeneous fault plane on which asperities fail by static fatigue, and showed that the calculated temporal behaviour of accelerating premonitory slip agrees with the observed time dependence of foreshocks. For precursory fault slips, Yamashita (1980) and Mikumo (1981) suggested independently that the cohesive properties of fault asperities, heterogeneous strength distribution, and a low stress level with respect to the average strength, could play a dominant role. A recent work of Das \& Scholz (1981) suggested that all phases of seismic phenomena including nucleation, precursory slow slips, foreshocks, multiple shocks, delayed multiple events, aftershocks, post-seismic slips etc., can be explained by the 
extension of shear crack and stress corrosion cracking. The results from these investigations are carefully examined in constructing our model.

The models we consider here are essentially based on a complete three-dimensional fault model (Miyatake 1980) in an infinite medium, but the present numerical calculations are made mainly on the quasi-three-dimensional fault as dealt with in the previous papers (Mikumo \& Miyatake 1978, 1979). The latter is an approximation to the above complete model, in the sense that the medium is bounded by a fixed boundary not very far from the fault plane, and hence that the absolute slip displacements in dynamic solutions are somewhat smaller than those expected from the complete calculations. However, this effect is not a serious problem in numerical simulations of seismic sequences, and hence the approximate model is again used for its shorter computation time.

In the present calculations, the external shear stress is applied parallel to the fault plane, and is assumed to be uniform at the nitial stage and to increase linearly with time due to the increase in tectonic loading. The entire fault plane is divided into a number of small fault segments with a unit dimension. We assume, as in the previous papers, that static and sliding frictional strengths are non-uniformly distributed on every fault segment. These nonuniformities will be simulated in various ways as described later.

The stress and displacement components at any point on the fault satisfy the equations of motion, and hence the dynamic motions of all fault segments due to the change of stress is specified by these relations under appropriate boundary conditions and a fracture criterion. All displacements at the extreme edges of the fault are fixed to be zero. This boundary condition is imposed here due to computational requirements for a finite area of the fault plane, and also seems natural in view of the existence of some geological boundaries around an actual fault. The fracture criterion we adopted here is again a finite stress criterion as in the previous papers, since this has been shown (Das \& Aki 1977) to be a good approximation to the criterion based on the critical stress intensity factor; once the applied shear stress exceeds static frictional strength at any fault segment, fault slip immediately occurs there, and is resisted by the sliding frictional strength. Thereupon, dynamical rupture is developed spontaneously, propagating up to the size specified by the distribution of shear stress and static strength. All these motions during the rupture propagation can be solved numerically as a dynamic problem by a finite difference scheme (Mikumo \& Miyatake 1978).

After the dynamical motion is completed, the initial stress on each of the fault segments drops to the level of sliding frictional strength there. In some cases for the present models, we consider a time-dependent stress recovery due to viscoelastic properties of fault gouge materials, which are assumed to be a linear standard solid, in addition to the tectonic stress increase. The temporal behaviour of the stress recovery has been given in a previous paper (Mikumo \& Miyatake 1979). On the other hand, the static frictional strength on the slipped segments is also assumed in these cases to drop after the rupture, and to recover with time in a logarithmic form due to creep at points of contact in re-locked portions. These behaviours have been suggested by laboratory friction experiments of rocks (e.g. Dieterich 1972). The above time-dependent recovery of shear stress and frictional strength may be a possible mechanism, but it would be possible to consider other types of mechanism such as time-delayed stress corrosion or weakening of the fracture strength.

The main physical parameters assumed in the present models are: (1) the rate of increase of tectonic shear stress, (2) the form of distributions of static and sliding frictional strengths and their spatial variations on the fault, (3) the rates of weakening and recovery of the fault strength, and (4) the relaxation time and relaxed elastic modulus of fault materials. The first two parameters may be of prime importance to the spatial and temporal variations of seismic sequences. The last two parameters will have appreciable effects on short-term post-seismic 
situations including aftershocks but may be omitted in most simplified simulations for a long time range.

For the spatial distribution of the frictional strengths on a real fault, we have actually no information from observations at this moment. However, there is some experimental evidence for the form of distribution from laboratory experiments for rock material; the tensile strength indicates a Weibull-type distribution for granite (e.g. Yamaguchi \& Nishimatsu 1967). We feel that the shear strength of intact rocks has a similar type of distribution, but it may be more natural for fault materials to have bi-modal or more complex strength distributions rather than represented by a single Weibull type. This is because the fault plane should have different degrees of contact; that is, tightly locked portions will have a high degree of contact due to indentation and ploughing asperities (Scholz \& Engelder 1976); however, the existence of fluid pore pressures will weaken the contact, and crushed fault gouge materials,will have a loose contact. Thus, there may be portions with strong and weak frictional strengths, although in this case the distribution is not always bi-modal but could

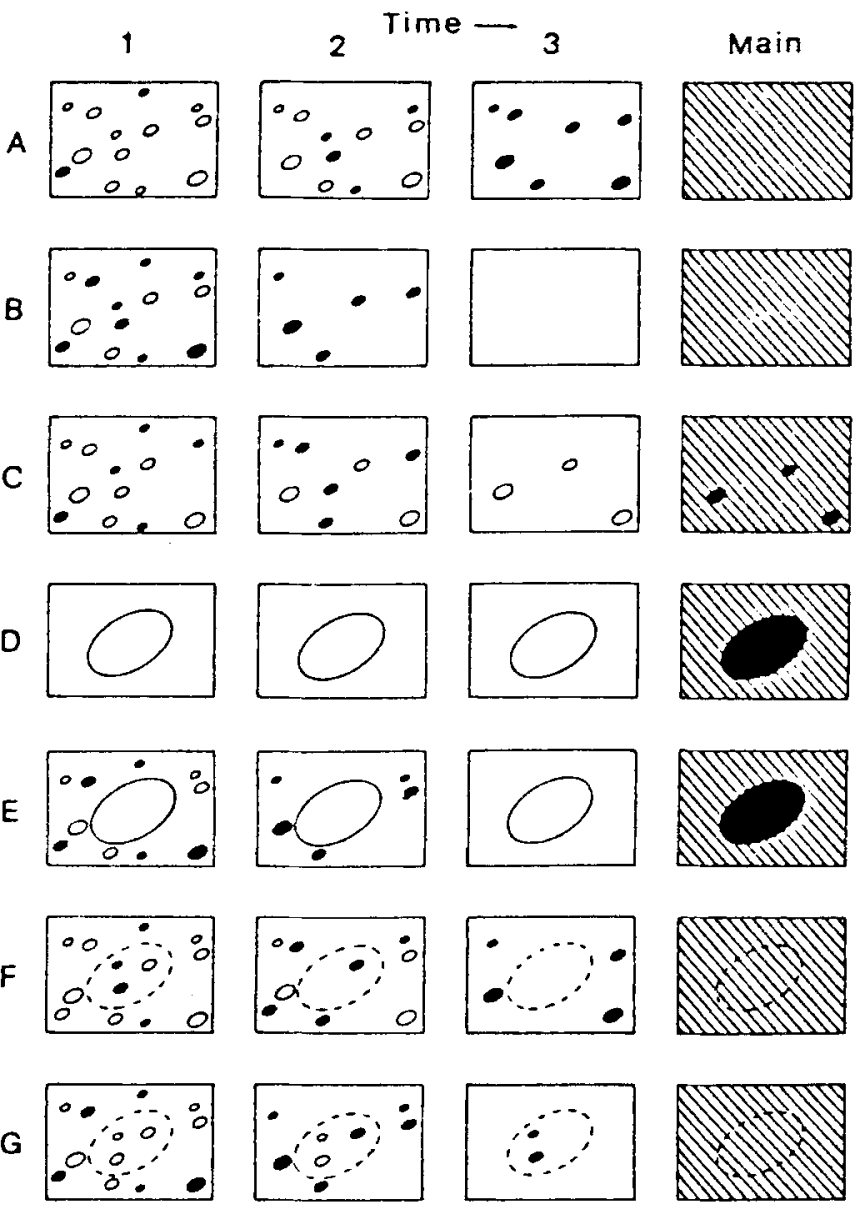

Figure 2. A schematic representation of several existing asperity models (after Utsu 1980). Rectangles indicate a fault surface which would break at the time of a main shock, solid ellipses show asperities which rupture at each of the indicated time steps, and open ellipses are those which have not been ruptured. 
A 1

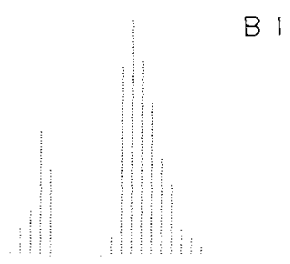

A 2

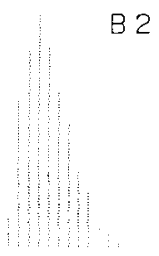

B 2

C 2

D 2
B 1

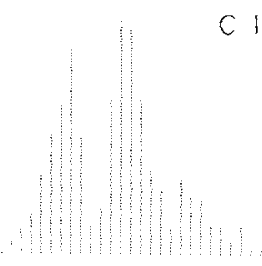

i

B)

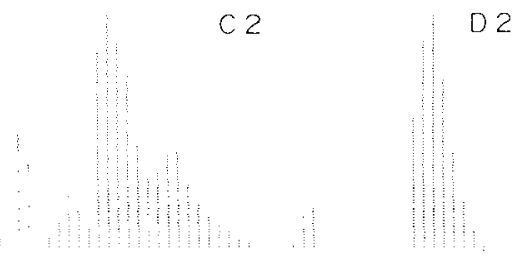

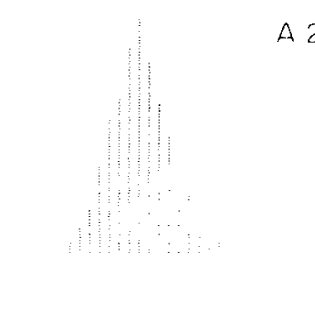

A 3

83

C 3

D 3
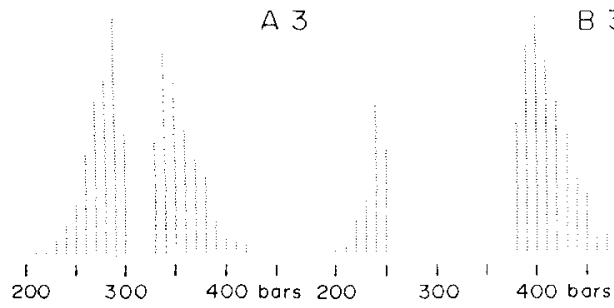

3
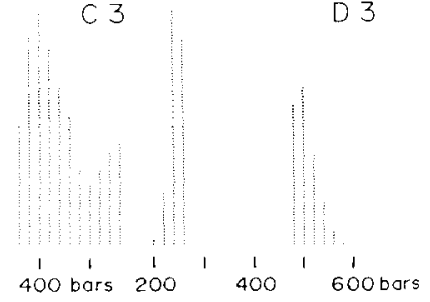

(a)
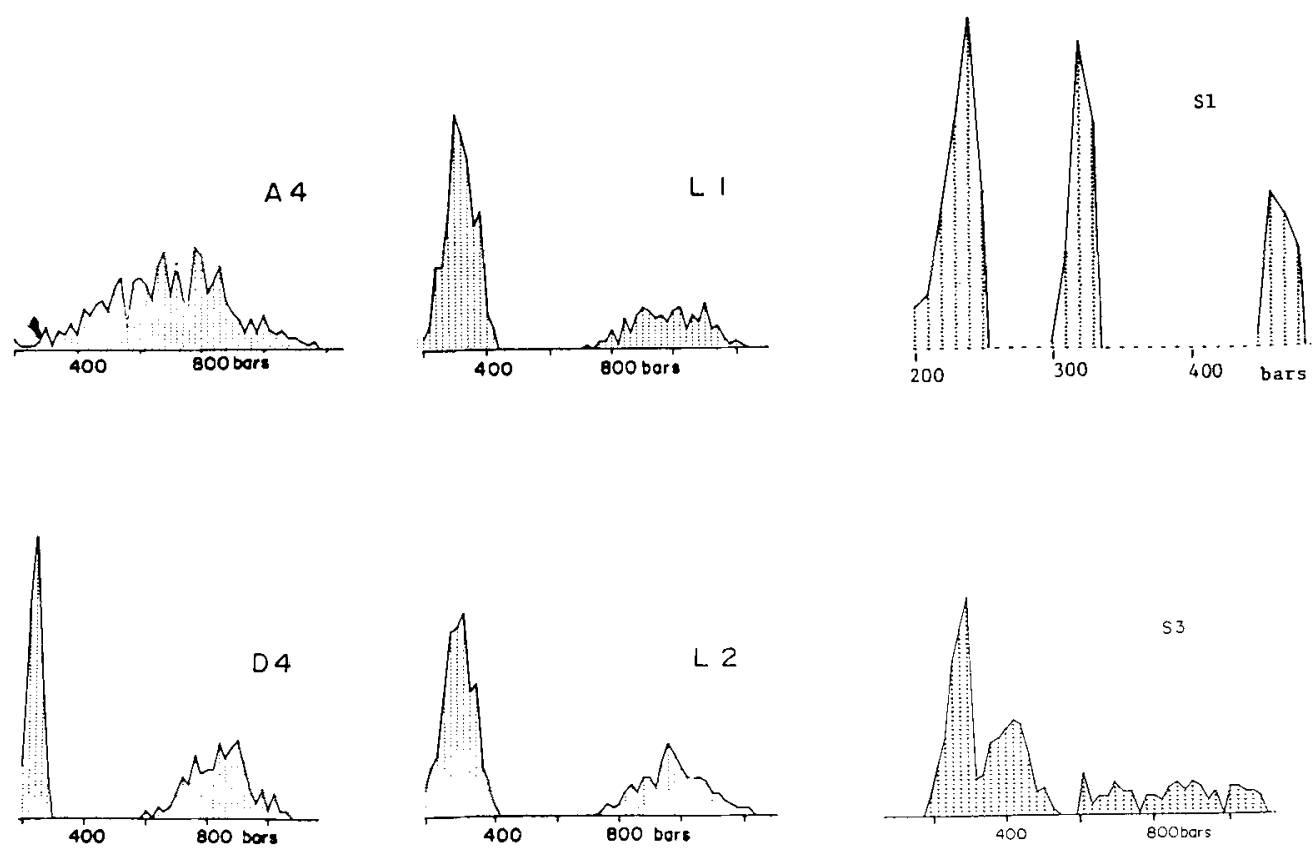

(b)

Figure 3. Various types of the assumed forms of distributions of static frictional strengths over the fault. For detailed features, see text. 
be multi-modal. Mizutani (1981, private communication) and Spetzler, Mizutani \& Rummel (1982) suggested, on the basis of laboratory experiments, that the fracture strength of rock materials could become bi-modal under increasing stress; this may be due to the formation of two different asperity sizes and of different strengths along two different symmetry axes of crystals, since the size of some asperities decreases and the initial orientation of some cracks tends to be aligned with the axis of maximum compressive stress, in response to the growth of microcracks as the stress increases. For the distribution of asperities, on the other hand, Utsu (1980) showed that there are three major types in existing asperity models so far proposed, which include: (1) many small-size asperities, (2) one or a few large-size asperities and (3) a mixture of small to large-size asperities, as schematically shown in Fig. 2. Taking all these situations into consideration, we assume the following types of distributions of the strength over the fault plane. These are:

(1) weakly to moderately non-uniform distributions, represented by a single modal, Gaussian type with different variances (models A1, A2, A3 and A4);

(2) moderately to heavily non-uniform distributions, including a number of small-size asperities with medium to high strengths, represented by a bi-modal Weibull type (models B1, B2, B3; D1, D2, D3 and D4);

(3) heavily non-uniform distributions including one or a few large-size asperities with medium to high average strengths, represented by a multi-modal Weibull type (modes $\mathrm{C}$, $\mathrm{C} 2, \mathrm{C} 3, \mathrm{~S} 1, \mathrm{~S} 2$ and $\mathrm{S} 3$ );

Table 1. Parameters specifying the fault models.

$\begin{array}{llllrlllllll}\text { Models } & \begin{array}{l}\sigma_{\mathbf{s}} \\ (\min )\end{array} & \begin{array}{l}\sigma_{\mathrm{s}} \\ (\mathrm{avw})\end{array} & \begin{array}{l}\sigma_{\mathbf{s}} \\ (\text { avs })\end{array} & \begin{array}{l}\sigma_{\mathbf{s}} \\ (\max )\end{array} & \begin{array}{l}\sigma_{\mathrm{d}} \\ (\min )\end{array} & \begin{array}{l}\sigma_{\mathrm{d}} \\ (\max )\end{array} & C_{\mathrm{d}} & \sigma_{\mathrm{s}} & \alpha & \begin{array}{l}\tau(\mathrm{s}) \\ (\times 10)\end{array} & L \times W \\ \text { A1 } & 200 & 290 & - & 329 & 150 & 247 & 1.00 & 0.0 & 1.00 & 1.555 & (1) \\ \text { A2 } & 200 & 300 & - & 390 & 150 & 292 & 0.88 & 6.0 & 0.70 & 1.555 & (1) \\ \text { A3 } & 200 & 290 & 340 & 429 & 150 & 322 & 0.88 & 6.0 & 0.70 & 1.555 & (1) \\ \text { A4 } & 200 & 700 & - & 1180 & 100 & 100 & 1.00 & 0.0 & 1.00 & \infty & (2) \\ \text { B1 } & 200 & 240 & 330 & 408 & 150 & 306 & 0.88 & 6.0 & 0.70 & 1.555 & (1) \\ \text { B2 } & 200 & 240 & 370 & 453 & 150 & 340 & 1.00 & 0.0 & 1.00 & 1.555 & (1) \\ \text { B3 } & 200 & 240 & 400 & 490 & 150 & 367 & 1.00 & 0.0 & 1.00 & 1.555 & (1) \\ \text { C1 } & 200 & 290 & - & 503 & 150 & 377 & 0.88 & 6.0 & 0.70 & 1.555 & (1) \\ \text { C2 } & 200 & 240 & - & 481 & 150 & 367 & 0.88 & 6.0 & 0.70 & 1.555 & (1) \\ \text { C3 } & 200 & 240 & - & 578 & 150 & 433 & 1.00 & 0.0 & 1.00 & 1.555 & (1) \\ \text { D1 } & 200 & 220 & 400 & 588 & 150 & 441 & 0.88 & 6.0 & 0.70 & 1.555 & (1) \\ \text { D2 } & 200 & 220 & 480 & 578 & 150 & 433 & 0.88 & 6.0 & 0.70 & 1.555 & (1) \\ \text { D3 } & 200 & 240 & 500 & 588 & 150 & 441 & 0.88 & 6.0 & 0.70 & 1.555 & (1) \\ \text { D4 } & 200 & 240 & 800 & 1180 & 100 & 100 & 1.00 & 0.0 & 1.00 & \infty & (2) \\ \text { S1 } & 200 & 340 & - & 552 & 150 & 414 & 0.75 & 3.0 & 1.00 & 1.555 & (1) \\ \text { S2 } & 200 & 350 & - & 606 & 150 & 455 & 1.00 & 0.0 & 1.00 & 1.555 & (1) \\ \text { S3 } & 200 & 300 & - & 1200 & 100 & 100 & 1.00 & 0.0 & 1.00 & \infty & (3) \\ \text { L1 } & 200 & 300 & 900 & 1200 & 100 & 100 & 1.00 & 0.0 & 1.00 & \infty & (2) \\ \text { L2 } & 200 & 300 & 900 & 1240 & 100 & 100 & 1.00 & 0.0 & 1.00 & \infty & (2)\end{array}$

$\sigma_{\mathrm{S}}(\min )$, minimum static strength; $\sigma_{\mathbf{S}}(\max )$, maximum static strength; $\sigma_{\mathrm{S}}(\mathrm{avw})$, average static strength on weaker fault segments; $\sigma_{\mathrm{S}}$ (avs), average static strength on stronger fault segments or asperities; $\sigma_{\mathrm{d}}$ (min), minimum sliding frictional strength; $\sigma_{\mathrm{d}}(\mathrm{max})$, maximum sliding frictional strength (the unit of strengths is given in bars). $C_{\mathrm{d}}$, weakening rate of static strength; $\delta \sigma_{\mathrm{s}}$, logarithmic recovery rate given by bars/one order of magnitude time interval; $\alpha$, relaxed elastic modulus; $\tau$, relaxation time; (1), $L \times W=40 \times 40 \mathrm{~km}$ (quasi-three-dimensional model); (2), $L \times W=30 \times 15 \mathrm{~km}$ (quasi-three-dimensional model); (3), $L \times W=$ $30 \times 15 \mathrm{~km}$ (complete three-dimensional model). 
(4) complex distributions including a specific shape barrier region with extremely high strengths, together with non-uniform strengths in the rest of the fault (models LI and L2).

The forms of the above distributions are shown in Fig. 3(a,b), and all the parameters specifying these distributions and other parameters are listed in Table 1 . These are only representative models, for each of which we have worked out more than five to ten cases with systematically varying these parameters.

\section{Model parameters in numerical calculations}

In the present calculations, the fault dimension $(L \times W)$ is taken as $30 \times 15 \mathrm{~km}$ in the complete three-dimensional model, and $40 \times 40 \mathrm{~km}$ or $30 \times 15 \mathrm{~km}$ in the approximate model, respectively. The total fault segments in these cases amount to 450 and 1600 respectively, the unit segment being taken as $1 \times 1 \mathrm{~km}$. The static frictional strength $\sigma_{\mathrm{s}}$ ranges between 200 and 1200 bar in the various distributions described above, including extremely high strength asperities in several cases. Sliding frictional strength $\sigma_{\mathrm{d}}$ is taken in most cases to be proportional to the static strength on each of the fault segments as in $\sigma_{\mathrm{d}} / \sigma_{\mathrm{s}}=0.75$ (Byerlee 1978), with a lowest value of 150 bar, and in several other cases it is assumed to be constant at 100 bar. The rate of weakening $C_{\mathrm{d}}$ of the static strength is assumed to be 0.88 and 1.0 (no weakening), and its logarithmic rate $\delta \sigma_{\mathrm{s}}$ is taken as 0.0 (constant), 3.0 or $6.0 \mathrm{bar} / \mathrm{time}$ increment in one order of magnitude. Also assumed is the coefficient of the relaxed elastic modulus $\alpha$ to be two cases of 0.7 and 1.0. The relaxation time $\tau$ is taken to be constant here as $1.555 \times 10^{7} \mathrm{~s}$ (half a year) or infinity, since we deal in this case with temporal variations of seismic activity over a very long time interval. A limiting case with $C_{\mathrm{d}}=1.0, \delta \sigma_{\mathrm{s}}=0.0$, $\alpha=1.0$ and $\tau=\infty$ corresponds to the simplest case when the weakening and recovery of frictional strengths and the viscoelastic recovery of shear stress are not taken into account. For about half of the cases studied here, we use this simplest assumption.

Table 2. Characteristic features of the selected models in Section 4.

\begin{tabular}{|c|c|c|c|c|c|}
\hline \multirow[t]{2}{*}{ No. } & \multirow[t]{2}{*}{ Model } & \multicolumn{2}{|c|}{ Strength distribution } & \multirow[t]{2}{*}{ Figures } & \multirow{2}{*}{$\begin{array}{l}\text { Observations } \\
\text { to be compared }\end{array}$} \\
\hline & & Mode & Features & & \\
\hline \multirow[t]{3}{*}{ (1) } & A1 & Single modal & Small variance & 3(a), 4(a), 6(a) & Case V \\
\hline & A2 & Single modal & Medium variance & $3(a), 4(b), 6(a)$ & Cases I, III, PB \\
\hline & A4 & Single modal & Large variance & $3(b), 5(a), 6(b)$ & Case IV \\
\hline \multirow[t]{2}{*}{ (2) } & B 1 & Bi-modal & $\begin{array}{l}\text { Small-size asperities } \\
\text { with medium strength }\end{array}$ & $3(\mathrm{a}), 4(\mathrm{c}), 6(\mathrm{a})$ & Cases I, II, PB \\
\hline & D4 & Bi-modal & $\begin{array}{l}\text { Moderate-size asperities } \\
\text { with high strength }\end{array}$ & $3(\mathrm{~b}), 5(\mathrm{a}), 6(\mathrm{~b})$ & Cases I, II, III \\
\hline \multirow[t]{2}{*}{ (3) } & C3 & Multi-modal & $\begin{array}{l}\text { Elliptic-shaped asperity } \\
\text { with medium strength }\end{array}$ & $3(\mathrm{a}), 4(\mathrm{~d}), 6(\mathrm{a})$ & Case DN \\
\hline & S1 & Multi-modal & $\begin{array}{l}\text { Elliptic-shaped asperity } \\
\text { with high strength }\end{array}$ & $3(\mathrm{~b}), 4(\mathrm{e}), 6(\mathrm{a})$ & Case DN \\
\hline \multirow[t]{2}{*}{ (4) } & L1 & Bi-modal & $\begin{array}{l}\text { Uniform asperity density } \\
\text { with a belt-like barrier }\end{array}$ & $3(b), 5(b), 6(c)$ & Case PB (DME) \\
\hline & L2 & Bi-modal & $\begin{array}{l}\text { Non-uniform asperity density } \\
\text { with a belt-like barrier }\end{array}$ & $3(\mathrm{~b}), 5(\mathrm{~b}), 6(\mathrm{c})$ & Case PB \\
\hline (5) & S3 & Multi-modal & $\begin{array}{l}\text { Square-shaped barrier on } \\
\text { a complete 3-D fault }\end{array}$ & $3(\mathrm{~b}), 5(\mathrm{a}), 6(\mathrm{~b})$ & Case DN \\
\hline
\end{tabular}

\section{Remarks:}

${ }^{\star}$ The observed results are discussed in Section 5. For case numbers, refer to the text. PB, great earthquakes along the plate boundary; DME, delayed multiple events; DN, doughnut pattern of seismicity. 
The initial shear stress due to tectonic loading is taken as 200 bar, and the rate of its increase is tentatively taken as $10-15$ bar $/ 3.15 \times 10^{7} \mathrm{~s}$ for computational convenience. The above rate may be one order of magnitude greater than its actual value from secular strain observations, but can be reduced to a reasonable rate if we take a longer time step than tentatively given below. The time step tentatively taken in the present calculations is

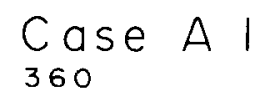

720

1080

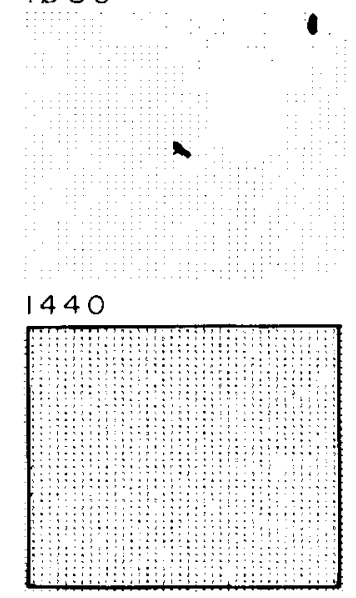

1980
2160

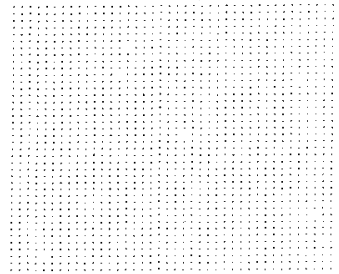

2520

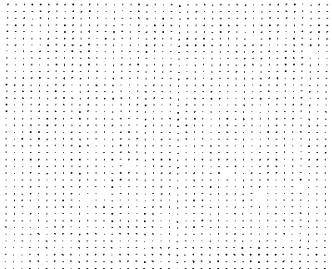

2700

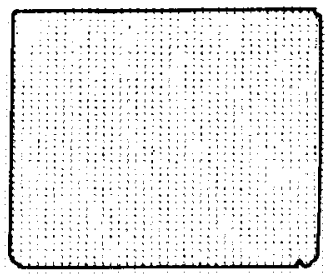

2880

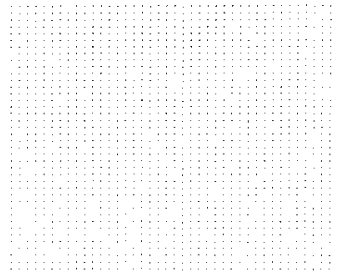

3060

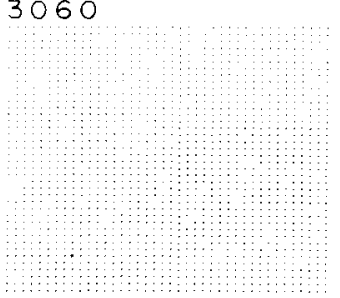

(a)

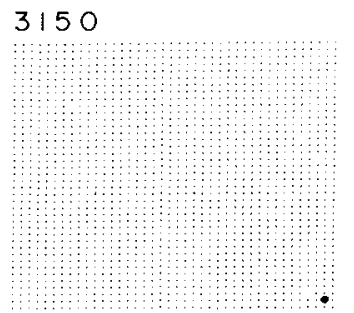

3240

3420

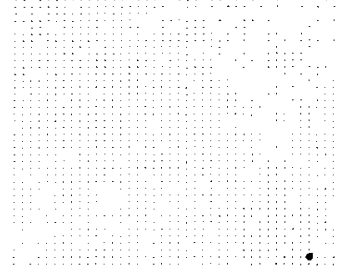

3600

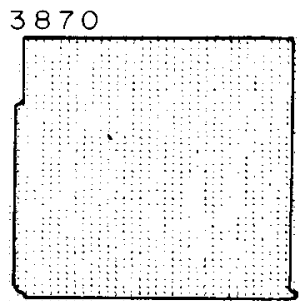

Figure 4. Calculated space-time seismicity patterns. Numerals on the left top on each of the patterns indicate the time step given in days. (a) model A1, (b) model A2, (c) model B1, (d) model C3, (e) model S1. Blacked-in areas indicate the shocks that occurred at the indicated time steps, and large outlined areas show a large shock with the rupture front reaching the edges of the prescribed fault plane. 
90-100 day, and can be scaled by the rate of increase of the tectonic stress. This means that if the rate of stress increase is taken as $1 / 10$ of the above value, then the calculated results will be for a time step 10 times longer. The time increment for dynamic solutions is taken as 0.05 or $0.10 \mathrm{~s}$. Other parameters not explicitly described here are taken as the same as in the previous papers.

Case $A 2$

1440

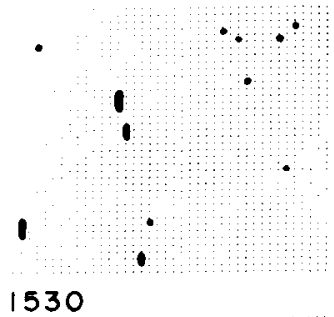

1530

1710

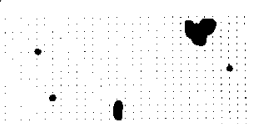

890

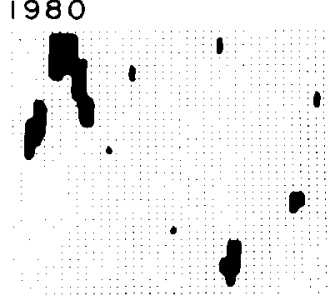

2070

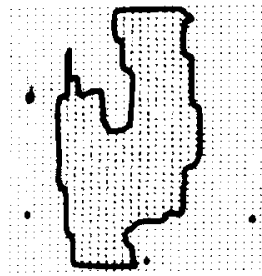

2160

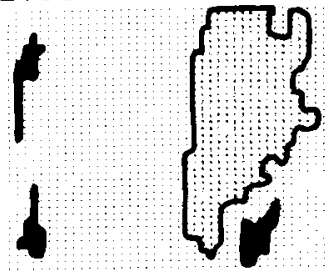

2250

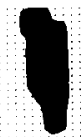

I

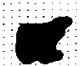

2340

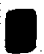

2430

9
2520
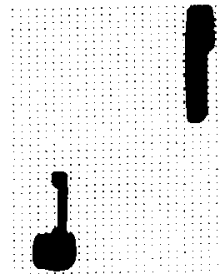

2610

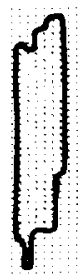

2700

I

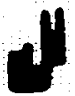

3060

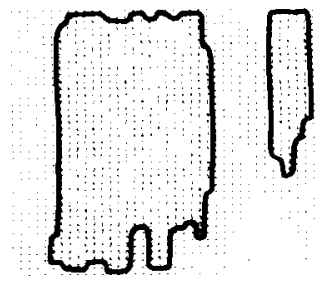

3330
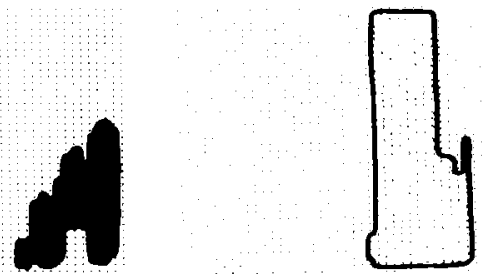

(b)

Figure 4 - continued 
4 Numerical simulations of space and time variations of seismicity patterns

In this section, several representative patterns are presented for the spatial and temporal variations of seismicity derived from numerical simulations on the present fault models. The characteristic features of the models selected here are summarized in Table 2. Also tabulated here are the reference numbers for the following discussion and the types of the observed temporal sequences that will be compared in Section 5 with the present results. Two

Case BI 1080

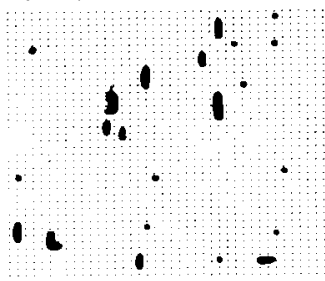

1350

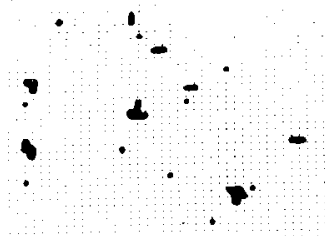

1440

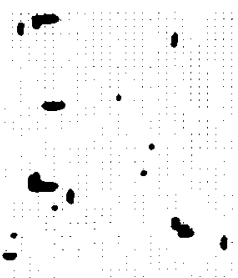

1530

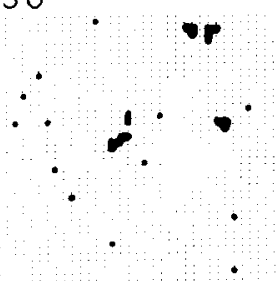

1620

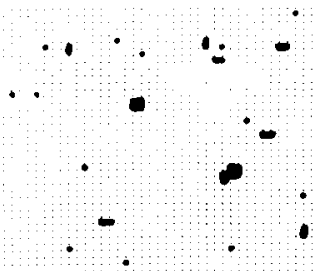

1800

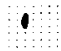

-
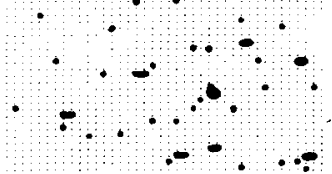

1890

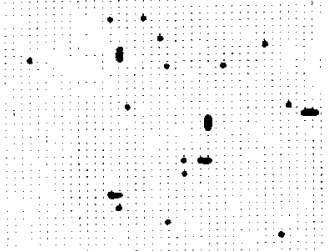

2610
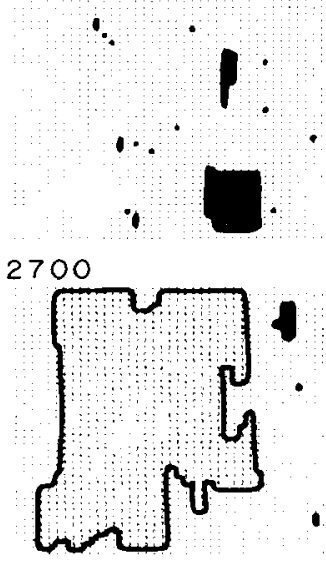

2790

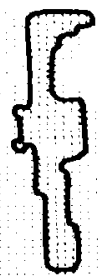

(c)

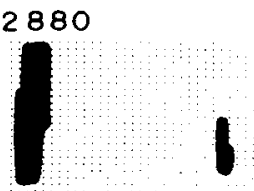

3150

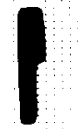

3420

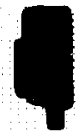

3780

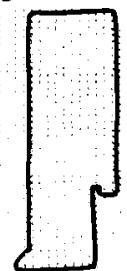

4050

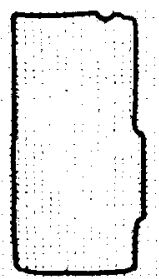

Figure 4 - continued 
different types of space-time plots are shown in this section to give the calculated results; one is two-dimensional patterns on the fault plane arranged in the order of discrete time steps (Fig. 4a-e), and the other is presented in a form of one-dimensional space $(x)$--time $(t)$ diagrams (Fig. 5a,b). The latter presentation is used for the cases with a large number of time steps, which are not adequately given in the former way. The temporal variations of these simulated seismicity patterns are shown in Fig. 6(a-c).
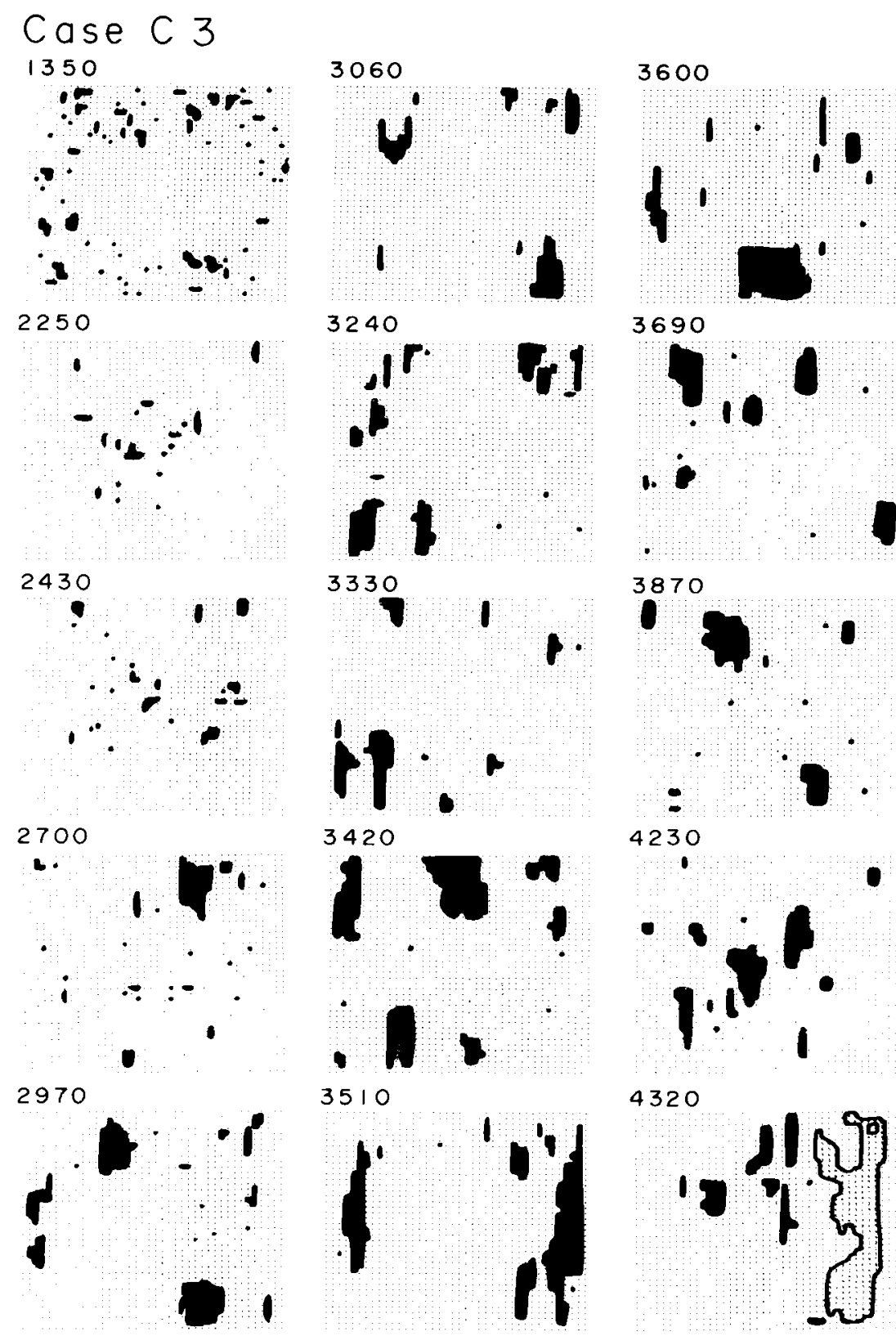

3510
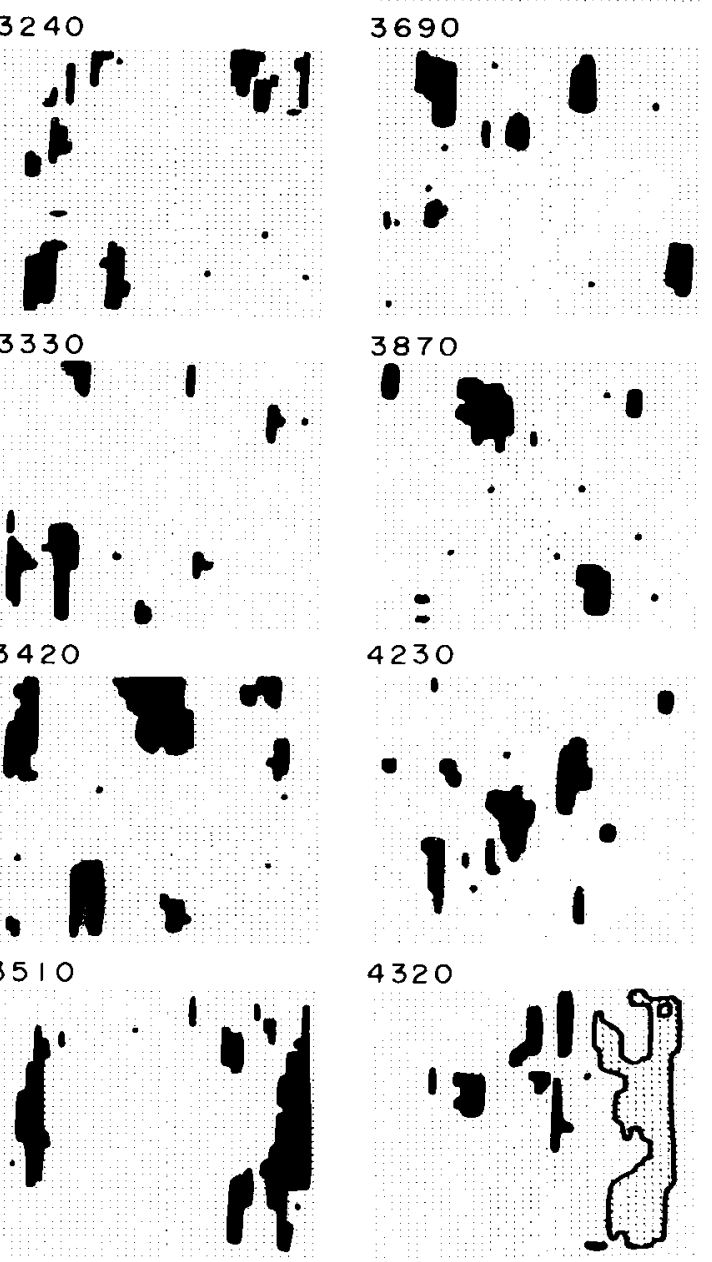

4320

(d)

Figure 4 - continued 


\section{Case $\mathrm{S}-1$}
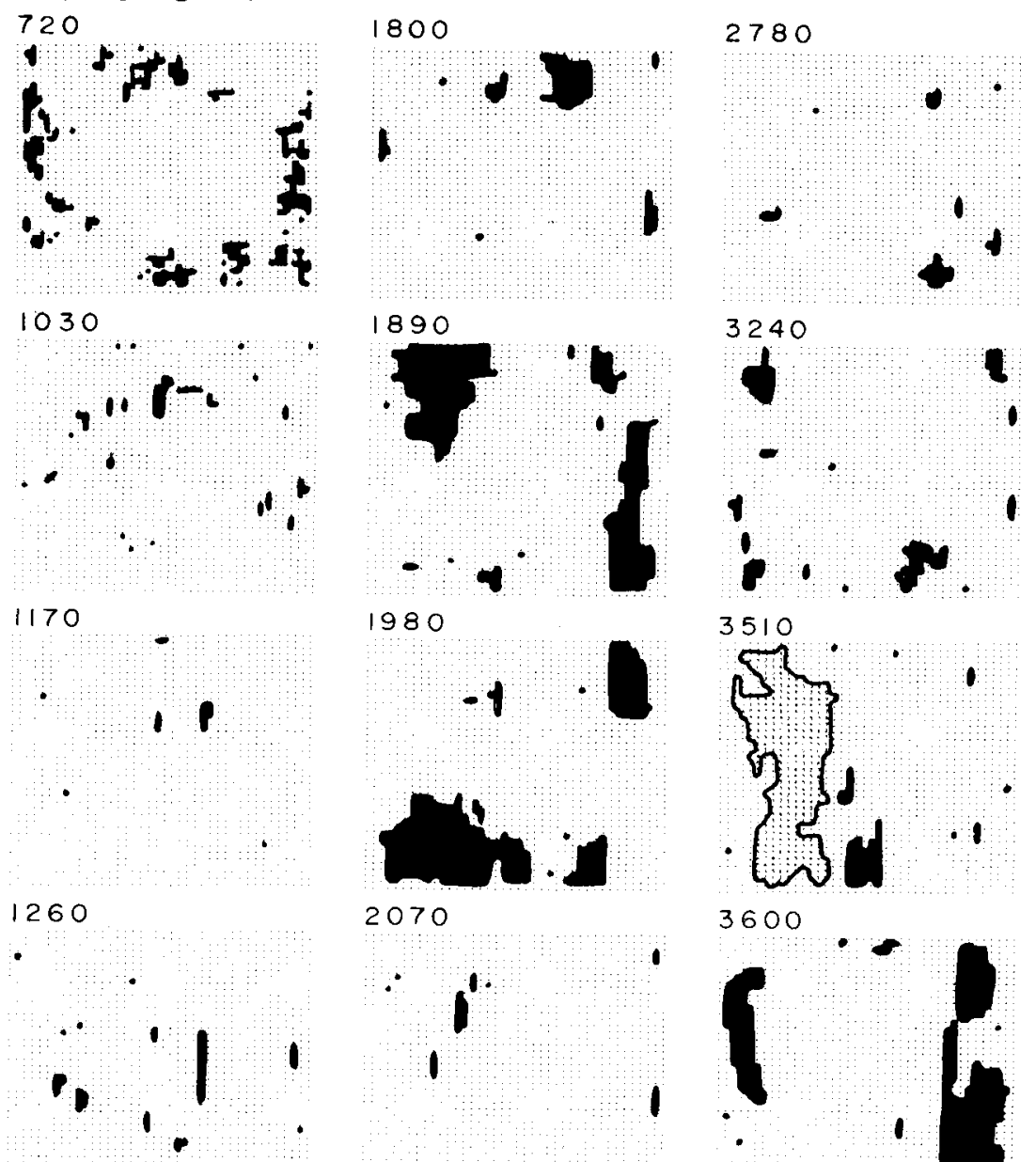

2070

1620
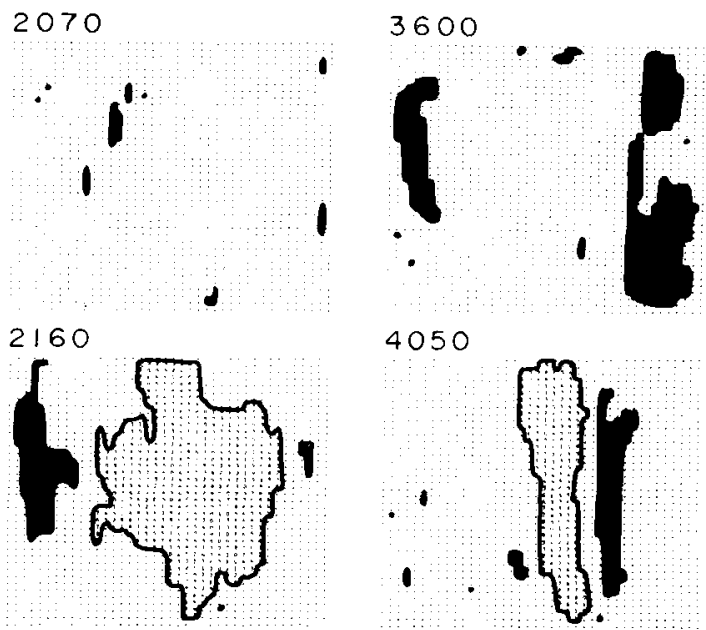

(e)

Figure 4 - continued

\section{4,1 SINGLE MODAL MODELS}

These models have weakly to strongly non-uniform strength distributions, which are represented by normal random Gaussian types with small to large variances. For model Al (Fig. 4a) with relatively homogeneous properties, only a few small shocks take place as the tectonic stress increases, and a large event suddenly occurs rupturing the entire fault plane without any precursory swarms or foreshocks after a long period of complete quiescence. 
After the main rupture there is again a long temporal gap, and a similar large event follows in the same way as above. Their recurrence times, which are slightly different, appear to be controlled simply by the rate of increase of the tectonic stress. In model A2 (Fig. 4b) with moderately heterogeneous properties, a large number of minor shocks take place successively at early stages, which may be regarded as a sort of foreshocks. A major shock occurs at 2070 day without pre-seismic quiescence, and another one follows at 2160 day within a


(a)

Figure 5. Left: Assumed spatial distribution of static frictional strengths over the fault. Hatched areas indicate asperities with high strengths, and a region enclosed by solid lines is assumed to be a barrier region with extremely high strengths. Right: one-dimensional representation of the space $(x)$-time $(t)$ seismicity patterns. $x$ and $y$ indicate the horizontal coordinates taken parallel to the two edges of a rectangle fault. $t$ is counted by the number of time steps, the interval of which is tentatively taken as 100 ' day. (a) models A4, D4 and S3, (b) models L1 and L2. 

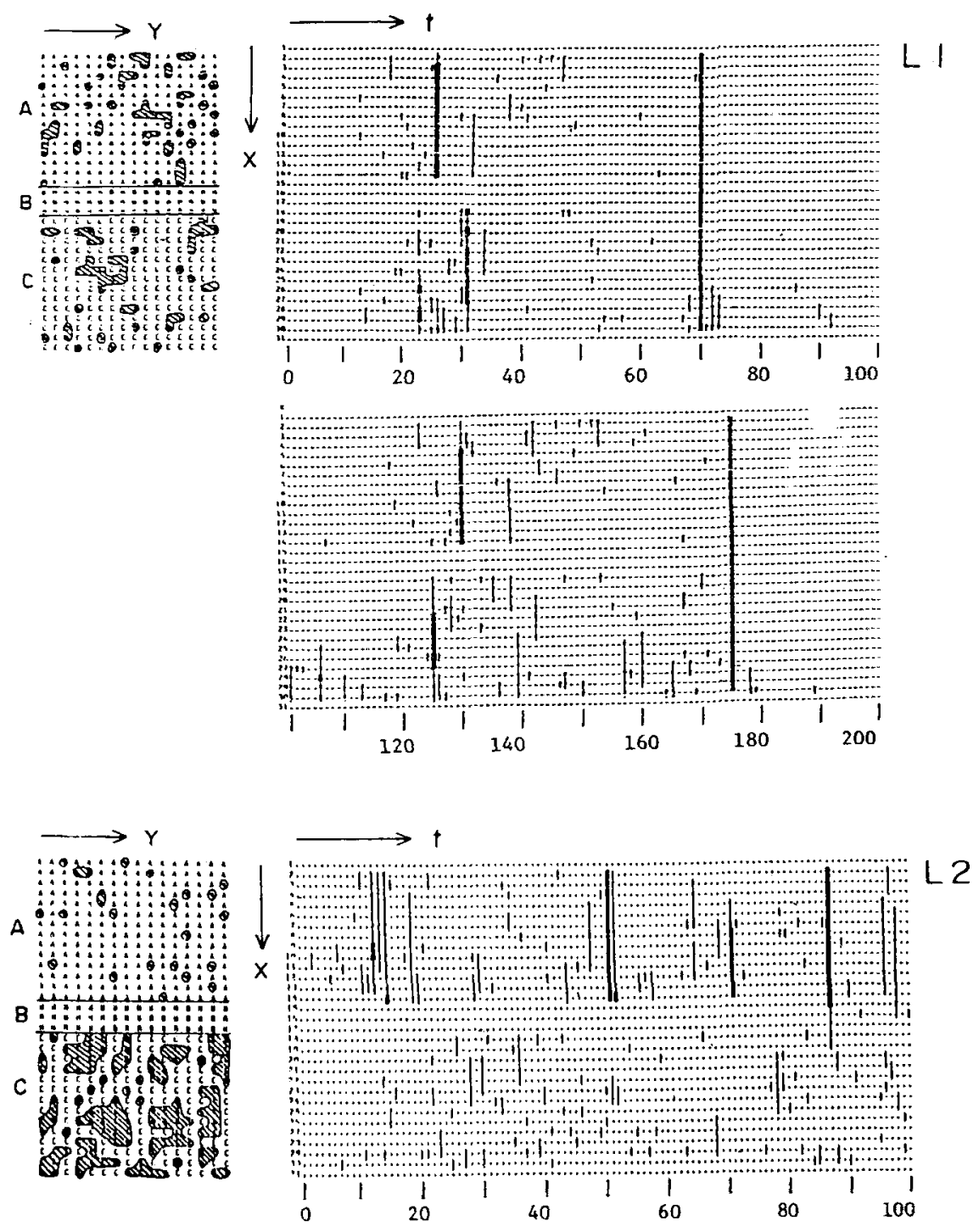

(b)

Figure 5 - continued

short time interval in the adjacent region. The recurrence of successive major shocks occurs at 3060 and 3330 day with a little longer time interval. These sequences appear to be strongly affected by the spatial variations of strengths. In model A4 (Fig. 5a), the strength distribution is assumed to be widely spread over $200-1180$ bar. In spite of its strongly heterogeneous properties, this model yields a rather simple temporal sequence, which exhibits a gradually increasing number of small shocks over a long period prior to a main event. This may be because it takes time to break widely spread strong heterogeneities, which would not allow the expansion of dynamic ruptures, generating only small shocks. A main event occurs at time step 91 covering the entire fault. There are, however, no precursory swarms or quiescence. These results indicate that as fault heterogeneities increase, small to moderate-size shocks tend to occur prior to a large event. 


\subsection{BI-MODAL MODELS}

The models include a large number of asperities over a moderately non-uniform background strength distribution, which is represented by a bi-modal Weibull type. For model B1 (Fig. 4c) with small-size asperities with medium strengths, small-scale weak portions are successively ruptured as minor shocks, and their activity becomes high during the period 1080-1800 day, which might be regarded as swarms extending over some time. A complete quiescence appears during the period between 1980 and 2520 day, and a large rupture follows with small foreshock activity. Some time after the main event, moderate-size shocks follow for some period, and there is a recurrence of large events at 3780 and 4050 day with somewhat different features. Model B3, which includes higher strength asperities, indicates somewhat different spatial patterns, although not shown here, but a similar temporal sequence to model B1. In model D4 (Fig. 5a) with moderate-size asperities with high strengths, there are two different series of activity; the edrlier one is successive ruptures of weaker segments and the latter is those of stronger portions, which leads to a main rupture covering the greater part of the entire fault. The former activity might be regarded as precursory swarms followed by a rather long period of low activity and quiescence, and the major event is
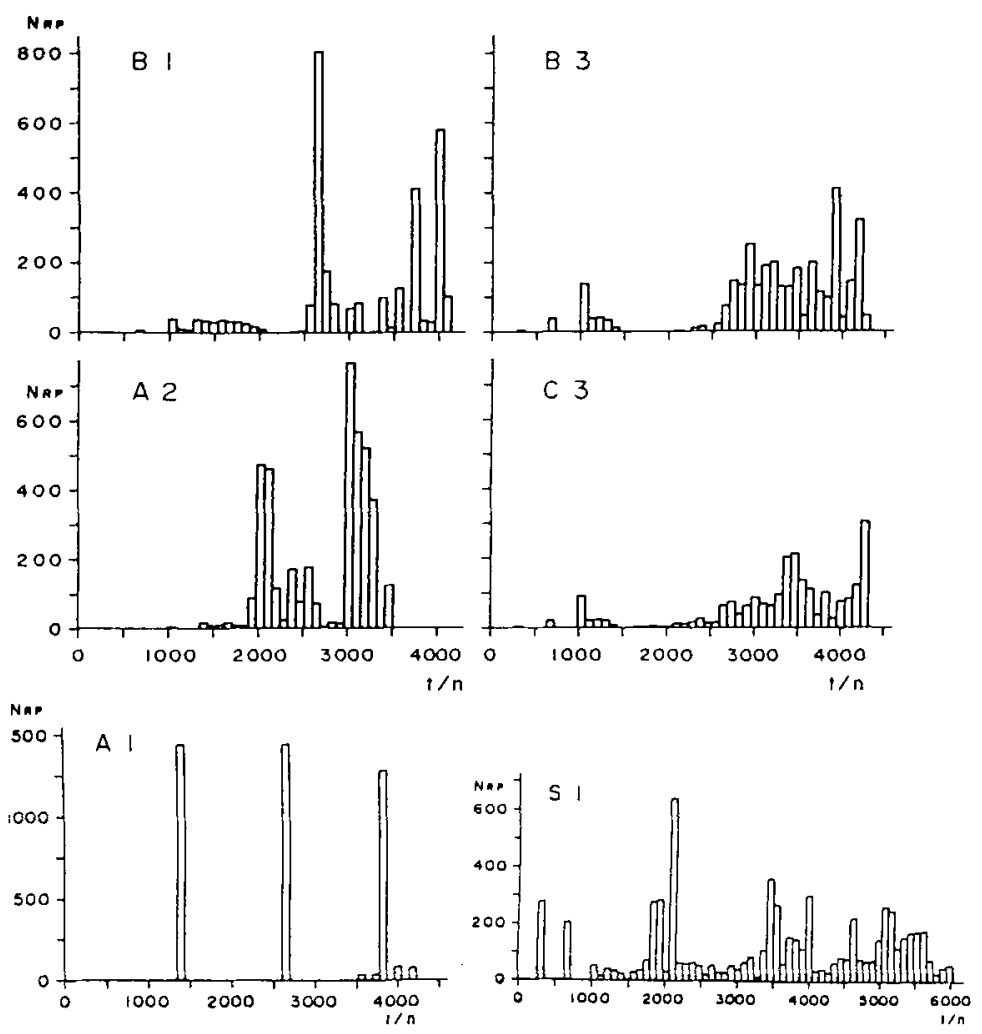

(a)

Figure 6. Temporal sequences of numerically simulated shocks on the assumed models. $N_{\mathrm{RP}}$ indicates the number of ruptured fault segments at each time step. (a) models B1, B3, A2, C3, A1 and S1. $t$ is given in days. (b) models A4, D4, S3. (c) models L1 and L2. Numerals in the abscissa indicate the number of time steps. 

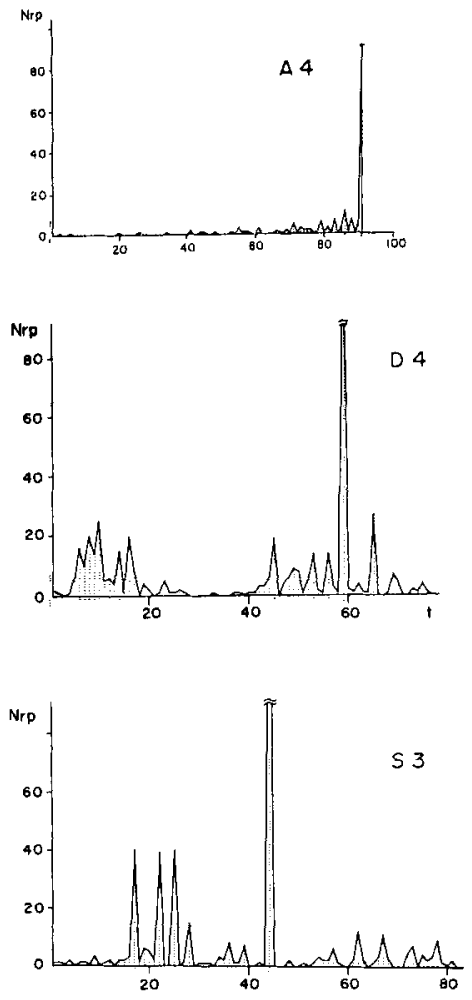

(b)
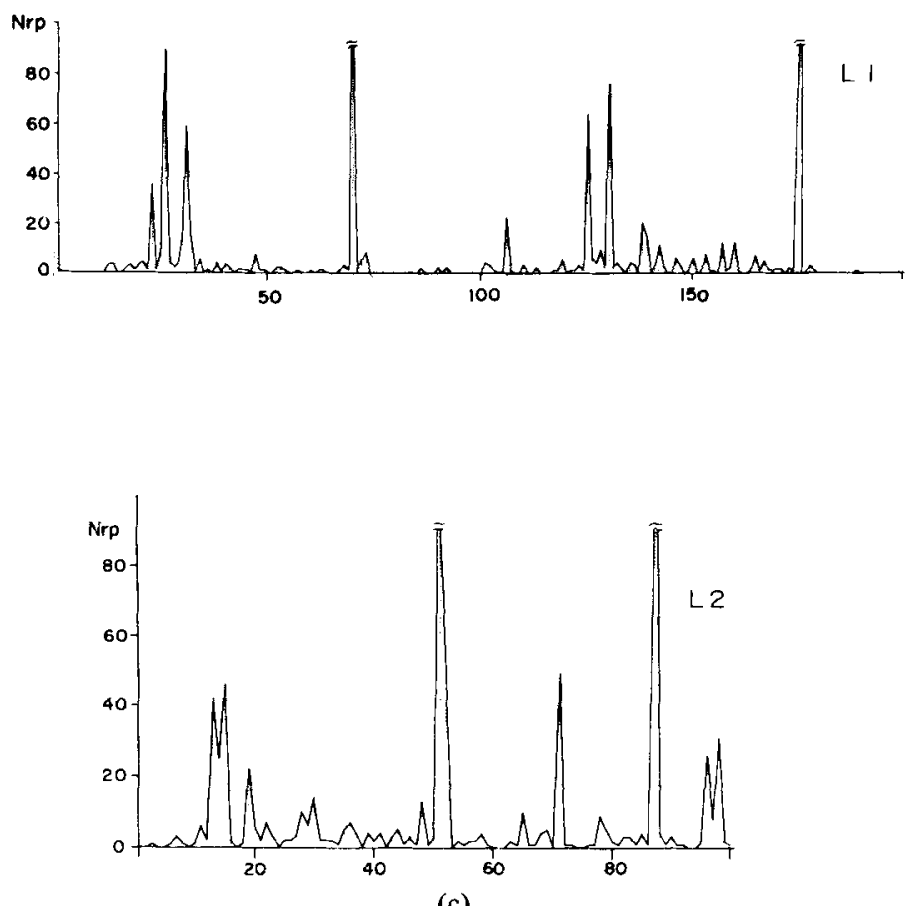

Figure 6 - continued 
preceded by foreshock activity. The above results indicate that the temporal behaviour appears essentially similar in these models, and that the period of quiescence depends mainly on the difference in the average strength between weaker segments and asperities.

\subsection{MULTI-MODAL MODELS}

These models assume multi-modal Weibull type strength distributions, which include a largesize elliptical-shaped asperity $(30 \times 20 \mathrm{~km})$ in the central part of the fault plane. A major difference between model C3 (Fig. 4d) and model S1 (Fig. 4e) is the average strength of the asperity region and the distribution density of weaker and stronger segments. In spite of these differences, similar spatial and temporal features are noticed up to some stages. At an early stage, small shocks take place by minor ruptures of weaker segments in the surrounding region, while the central asperity fegion remains quiet. This state is followed by a long period of complete quiescence for some time all over the fault. After this time, a number of minor shocks tend to occur in a part of the centrai asperity, indicating some clustering during a period, whereas the peripheral region becomes less active. This clearly shows a doughnut pattern of seismicity. Seismic activity with moderate-size shocks again moves towards the surrounding region, leaving the central region almost quiet. These patterns alternately appear in model C3. In model S1, a large event occurs at 2160 day covering a greater part of the central asperity region and a part of its surroundings. Activity after the major event becomes high in the surrounding region and large-scale shocks take place there at 3510 and 3600 day with a short time interval. Although the time series in the two models are quite similar to each other, pre-seismic swarms and quiescence are less evident in model S1.

\subsection{MODELS WITH A BELT-LIKE BARRIER REGION}

These models include a belt-like barrier region with high strengths in the central part of the fault, dividing the fault plane into two sections. The distribution density of asperities with medium strengths in the two sections is assumed to be equal at 20 per cent in model $\mathrm{Ll}$ (Fig. 5b), while it is appreciably different, 10 per cent in region $A$ and 50 per cent in region $\mathrm{C}$ in model L2 (Fig. 5b). In model L1, foreshock activity starts to occur, except in the barrier region, and then large events take place on the two divided sections at slightly different time steps. It can be seen that a main event rupturing the central barrier region and covering the entire fault occurs after a long period of low activity, but there is no precursory swarm or foreshock activity preceding the main rupture. A long period of complete quiescence follows the main event. As time goes on, the recurrence of these sequences appears over a long time range. In model L2, on the other hand, moderate to large-scale events take place repeatedly in region A with some foreshock activity, but only minor to moderate-size shocks successively occur without any regularities in region $\mathrm{C}$. The central barrier region remains quiet for a long time, and is broken at some time later when a large event recurs in region $\mathrm{A}$, possibly due to high stress concentration towards the barrier. The above results indicate that the distribution density of asperities in the two divided sections has remarkable effects on the seismicity patterns there.

\subsection{A COMPLETE 3-D MODEL}

We investigate here the space and time variations of seismic sequences on the basis of a more complete three-dimensional fault model (Miyatake 1980), to test the validity of the fore- 
going results from the quasi-three-dimensional model. In model S3 (Fig. 5a), we assume the existence of a large-scale square-shaped barrier region in the central part of the fault plane, with small-size asperities with high strengths in the rest of the fault. The form of strength distribution is multi-modal. The space-time pattern clearly shows that seismicity with minor to moderate-size shocks becomes active in the surrounding region for a long duration up to time step 30, while the central barrier region remains in complete quiescence, indicating a typical doughnut pattern of seismicity. As time goes on to step 40 , foreshock activity starts in a part of the barrier region, whereas the surrounding region becomes quiet. A large rupture occurs at time step 44 , covering the central region. After the main event, the entire fault tends to be quiet and minor shocks start to take place again in the surrounding region. These features are essentially similar to those for models C3 and S1. The time sequence of these events clearly shows a typical pattern including precursory swarms, quiescence and foreshocks prior to a main event.

\subsection{COMPARISON WITH EXPERIMENTAL STUDIES}

The above numerical results are compared here with some laboratory experimental studies. Mogi $(1963 a, b)$ has shown that the fracture of some model materials under a gradually increasing stress yields three typical patterns for a sequence of elastic shocks, depending on the degree of heterogeneities of applied stress and the structure of materials. The results indicate that; if the material is homogeneous and the stress is uniformly applied, a main shock occurs without any preceding shocks; this result is consistent with that for our single modal model A1 with a small variance; when the material has moderately heterogeneous structure and the stress is non-uniformly applied, small shocks gradually increase prior to a main event; this feature seems consistent with that for the single modal model A4 with a large variance; when the material is extremely heterogeneous and/or the applied stress is concentrated to a considerable extent, elastic shocks take place in a swarm without principal shock; there are no corresponding numerical simulations to be compared, but this state would be realized if the fault strengths are almost uniformly distributed in a specific range. Thus, a part of the numerical results obtained here seem qualitatively consistent with the laboratory studies, although the conditions are quite different; the experiments were made for solid materials under a compressive stress, while our numerical simulations are for the fault surface subjected to shear stresses.

Table 3. Different types of precursory changes of observed seismicity.

\begin{tabular}{|c|c|c|c|c|c|c|c|c|c|}
\hline Case & Normal stage & \multicolumn{7}{|c|}{ Precursory stage } & Main event \\
\hline 1 & $\begin{array}{l}\text { Normal background } \\
\text { seismicity }\end{array}$ & - & $\begin{array}{l}\text { Precursory } \\
\text { or clusterin }\end{array}$ & $\begin{array}{l}\text { swarms } \\
\text { hg }\end{array}$ & $\begin{array}{c}- \text { Pre-seismic } \\
\text { quiescence }\end{array}$ & $\begin{array}{l}\text { - Foresh } \\
\text { or clust }\end{array}$ & $\begin{array}{l}\text { ocks } \\
\text { eruig }\end{array}$ & - & Main shock \\
\hline $\mathbf{I}^{\prime}$ & $\begin{array}{l}\text { Normal background } \\
\text { seismicity }\end{array}$ & - & $\begin{array}{l}\text { Precursory } \\
\text { or clusterin }\end{array}$ & $\begin{array}{l}\text { swarms } \\
\text { ig }\end{array}$ & $\begin{array}{l}\text { - Pre-seismic } \\
\text { quiescence }\end{array}$ & $-\quad-$ & - & - & Main shock \\
\hline II & $\begin{array}{l}\text { Normal background } \\
\text { seismicity }\end{array}$ & - & $-\quad-$ & - & $\begin{array}{l}\text { - Pre-seismic } \\
\text { quiescence }\end{array}$ & $\begin{array}{r}- \text { Foresh } \\
\text { or clus }\end{array}$ & $\begin{array}{l}\text { ocks } \\
\text { tering }\end{array}$ & - & Main shock \\
\hline II' & $\begin{array}{l}\text { Normal background } \\
\text { seismicity }\end{array}$ & - & - & - & $\begin{array}{r}\text { - Pre-seismic } \\
\text { quiescence }\end{array}$ & - & - & - & Main shock \\
\hline III & $\begin{array}{l}\text { Normal background } \\
\text { seismicity }\end{array}$ & - & $\begin{array}{l}\text { Precursory } \\
\text { or clusterin }\end{array}$ & $\begin{array}{l}\text { swarms } \\
\text { lg }\end{array}$ & - Normal seisr & micity & - & - & Main shock \\
\hline IV & $\begin{array}{l}\text { Normal background } \\
\text { seismicity }\end{array}$ & - & - & Gradua & $11 y$ increasing $\mathrm{s}$ & eismicity & & - & Main shock \\
\hline V & No ba & ckg & und seismici & ity or pr & ecursory pheno & omena & & - & Main shock \\
\hline
\end{tabular}




\section{Discussion}

Now we discuss here various observations on the spatial and temporal variations of seismicity prior to major earthquakes, in comparison with the foregoing results from numerical simulations on heterogeneous fault models.

The temporal behaviours of the seismicity patterns observed before large earthquakes are summarized in a comprehensive review by Kanamori (1981). Here, we classify the observations into the following categories, as given in Table 3 , although this classification is only tentative for easy comparison with the numerically simulated results. Case I corresponds to the typical patterns proposed by Evison (1977b), and case II has similar but slightly different patterns of seismicity as suggested by Katsumata \& Yoshida (1980). A major difference between cases I and II lies in whether precursory swarms occur or not before a period of quiescence. These two representative cases are schematically illustrated in Fig. 7. The difference between cases I and $\mathrm{I}^{\prime}$ and between cases II and II' is whether foreshock activity precedes a main shock or not after the period of quiescence. Case III includes precursory swarms but no distinct quiescence or foreshocks, and case IV corresponds to the case when only increasing seismicity has been observed over a long period before a main shock. In case $\mathrm{V}$, which is rather unusual, no indications have been observed prior to a large earthquake. In the following discussion, a magnitude threshold and a time-scale would be needed for the observations, in comparing them with the numerical results. A simple estimate from the numerical calculations with appropriate fault dimension and slip displacements shows that it may be adequate to take a magnitude range between 4.0 and 7.5 and a maximum time interval of about $25 \mathrm{yr}$. However, these values can be scaled in numerical simulations to accommodate the observations with larger or smaller ranges.

It has sometimes been experienced as in case $\mathrm{V}$ that a large earthquake appears to have taken place suddenly in a quiet zone without any precursory indications anywhere around the rupture zone, although this might be due to poor coverage of observed data in some cases. The Sitka, Alaska earthquake of 1972 seems to be one of these examples with no precursory changes of seismicity (Kelleher \& Savino 1975). The sudden occurrence of this type of large earthquake may be simulated by model A1, which does not yield any significant precursory signals. This comparison suggests that this type of earthquake takes place in a fault zone with relatively homogeneous strengths, and also that there could be a recurrence in a future in the same way as above. If this is the case, it would be extremely difficult to make predictions for these earthquakes from the variations of seismicity patterns.
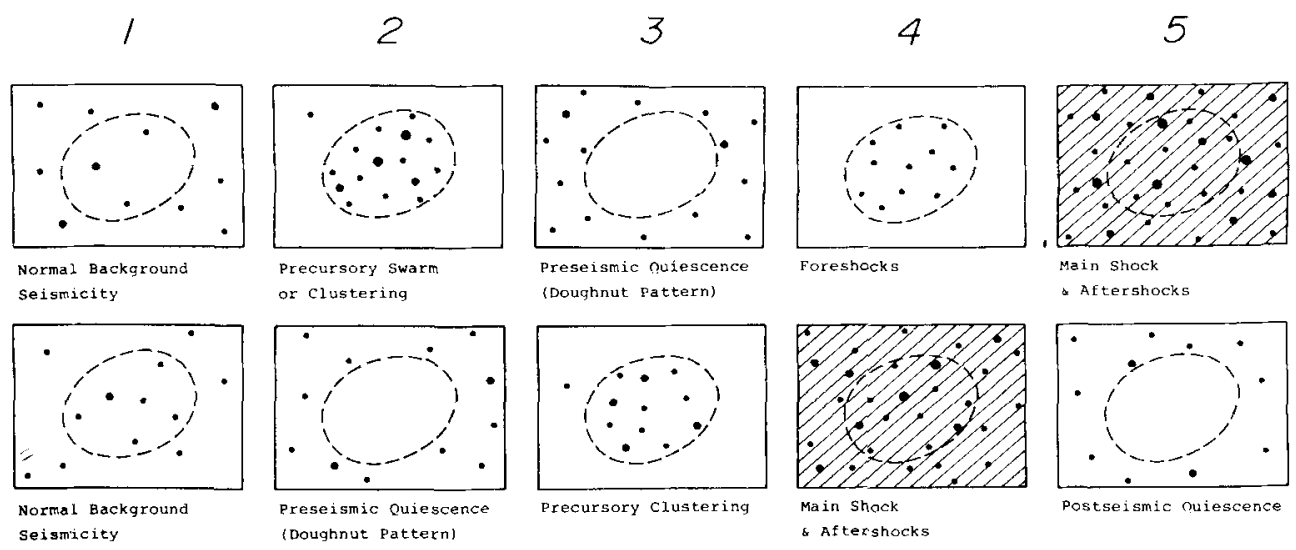

Figure 7. Schematic representation of two types (cases I and II) of observed seismicity patterns. 
Case IV may be applied to large interplate earthquakes in the Alaska-Aleutian regions. The 1958 and 1964 Alaska, 1965 Rat Island and 1957 Fox Island earthquakes were preceded by gradually increasing seismic activity over many years before the main shocks (Kelleher \& Savino 1975). The 1923 Kwanto earthquake in Japan appears to belong to this group (Sekiya 1977). The pre-seismic activity might be regarded as a sort of foreshocks but is not accompanied by distinct swarms and quiescence. The temporal behaviour of these sequences appears to be simulated by model A4. This might suggest that the fault zone in these regions could be extremely heterogeneous on a small scale but would not include large-size asperities.

There are several earthquakes as in case III that included only precursory swarms or high activity some time before the main shock but did not indicate any other phenomena. Several intraplate earthquakes in Japan and New Zealand, such as the 1948 Fukui and 1968 Inangahua earthquakes appear to belong to this group (Sekiya 1977; Evison 1977b). However, these types might be regarded as slight modifications from cases $\mathbf{I}$ or $\mathbf{I}^{\prime}$, if we could think that a small number of shocks occurred in a period of quiescence in case $I^{\prime}$ or that foreshock activity in case I was not concentrated just before the main shock but spread over the period of quiescence. If this is true, this type of earthquake might be accounted for by some version of the numerical simulations appropriate to cases $\mathbf{I}$ or $\mathbf{I}^{\prime}$. Except for the above three unusual types of seismic sequences, many of the other earthquakes may be classified into cases $I$ and Il or their modifications, cases I' and II'.

It is evident in Kanamori's (1981) review that several intraplate earthquakes such as the 1965 Borrego Mountain, 1979 Imperial Valley, 1952 Kern County and 1971 San Fernando earthquakes, and two large interplate earthquakes of 1963 and 1969 in the Kuriles have the typical features of case I, which consists of precursory swarms, quiescence, foreshocks and a main event, while some other intraplate earthquakes inclu ding the 1969 Gifu and 1974 Off-Izu earthquakes in Japan and several New Zealand earthquakes, and also a few interplate earthquakes such as the 1971 Kamchatka and 1968 Off-Tokachi earthquakes, appear to correspond to case I' because of lack of foreshock activity. A close comparison in dicates that the temporal sequences of these earthquakes may be well explained by numerical simulations on models B1, B3 and D4. The results would suggest that the fault zone of these earthquakes could have moderately to heavily heterogeneous properties with a number of high-strength asperities, if our present models are correct. The difference between cases I and I' may result from the degree of concentration of higher strengths in these models; that is, if high strengths are spread over a somewhat wide range as in model D4, foreshock activity would continue for some period, but otherwise there could be no foreshocks prior to the main event. Precursory clustering of seismicity around the zone of an impending main earthquake could also be explained by a concentration of asperities with medium strengths there.

The other typical type of seismic sequence is case II or II'. A few intraplate earthquakes such as the 1964 Niigata and 1966 Parkfield earthquakes, and several large interplate earthquakes including the 1960 Chilean, 1965, 1968 and 1978 Oaxaca, 1976 central Aleutian, 1952 Kamchatka, 1973 Kurile, 1952 Off-Tokachi, 1973 Off-Nemuro and 1938 OffFukushima earthquakes, seem to be classified as case II in which no clear precursory swarms or high activity have been observed before a period of quiescence; intraplate earthquakes such as the 1975 Hawaii and 1978 Shimane earthquakes, and several interplate earthquakes including the 1933 Sanriku, 1944 Tonankai, 1946 Nankaido, 1973 Kamchatka, and 1966 and 1974 Peruvian earthquakes appear to belong to case II' $^{\prime}$ which lack both swarms and foreshocks (Kanamori 1981). It seems likely that the temporal behaviour of these two cases can be simulated with some modifications of model B1 or other bi-modal models. If the strengths of weaker fault segments in model B1 or D4 are more widely distributed, seismic 
activity would not appear as a form of precursory swarms but would reduce to normal seismicity over a long period before quiescence as in case II. Foreshock activity also would

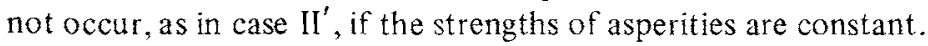

It has been reported (e.g. Mogi 1969; Yamashina \& Inouye 1979) that some of the earthquakes belonging to cases II or II' and a few belonging to case I were preceded by doughnut patterns of seismicity on local or regional scale. It appears that these observations on local scales might be accounted for by some multi-modal models such as models C3, S1 and S3, which have a large-scale asperity region with medium to high strengths. These models yield complicated space and time patterns of seismicity, composed of a long quiet period, swarms, quiescence and high activity in the central region, and high activity, quiescence and an active period followed by lesser activity in the surrounding regions. These alternate patterns in the two regions could provide a possible explanation to this type of observed seismic sequences such as described by Ishida \& Kanamori $(1978,1980)$ for two California earthquakes. There are some questions, however, whether regional-scale doughnut patterns over a wide area, such as described by Mogi (1969), could be accounted for by the present model.

It is well known that great interplate earthquakes in several subduction zones tend to occupy a large rupture zone extending for a few hundred kilometres along a plate boundary. It has also been shown that extremely large earthquakes ruptured at some other time, the entire source region covering the above zones. The successive occurrence in two adjacent 1946 Nankaido earthquakes and the 1854 Ansei I and II earthquakes (Ando 1975; Utsu 1977), the case of the 1957 central Aleutian and 1965 Rat Island earthquakes (Sykes 1971; Sykes et al. 1980), and the case of the Solomon Island earthquakes (Lay \& Kanamori 1981). It has also been shown that extremely large earthquakes ruptured at some other time, the entire source region covering the above zones. The successive occurrences in two adjacent regions within a short time interval may be explained by rapid stress concentration around the boundary of the two regions due to the rupture of the first earthquake. It appears that this phenomenon might be simulated to some extent by models $A 2$ and $B 1$, but the recurrence of the large earthquakes rupturing the entire plate boundary, together with the delayed multiple events, may be more adequately modelled by model Ll with a barrier region between the two source areas. However, the lag in their occurrence times needs to be more carefully investigated. The above phenomena have also been explained in other ways: by the extension of subcritical crack growth (Das \& Scholz 1981), by different distribution of asperities and their interactions (Lay \& Kanamori 1981) and with different average strengths in two regions (Miyatake 1982).

Premonitory slow fault slips at depths are another type of precursory seismic phenomenon observed before some large earthquakes. It has been shown by laboratory experiments (e.g. Dieterich 1979) that heterogeneous properties of fault surface are responsible for the precursory slips, and also suggested from numerical experiments (e.g. Mikumo 1981) that strongly cohesive properties of fault asperities as well as heavily non-uniform strength distribution could generate slow fault slips under a low level of applied shear stress. These precursory fault slips could sometimes trigger normal shocks, and sometimes could be triggered due to stress concentration by normal shocks in adjacent fault segments, even if a part or some localized portions of the fault have the above properties. If this is the case, it seems possible that normal shocks and slow slips could take place together or independently in a sequence of seismic activity over a fault zone.

Finally, we briefly mention substantial properties of asperities. We have the standpoint that fault asperities are manifestations of the portions with high degree of contact, such as by indentation and ploughing (Scholz \& Engelder 1976) between two sides of the fault surface, that is, tightly coupled strong portions, and hence should have high frictional 
strengths. Katsumata \& Yoshida (1980) and Lay \& Kanamori (1981) argued that mechanical coupling conditions between the subducting oceanic lithosphere and the overriding continental plate would play an important role in seismic activity in an eventual epicentral region; a strongly coupled state, if it results from a large area of contact and high frictional strength, would correspond to a large-size asperity. The stress state on the contact zone will be more or less uniform, and hence yield a seismic gap, although the mechanism of its temporal variations is not necessarily clear. Besides the coupling conditions, geometrical configurations such as irregular shape, local bending or discontinuities of the fault may be regarded as apparent asperities or barriers with high strengths. The existence of a barrier region has been actually characterized by the extent of surface faulting, the stopping of rupture propagation, and the spatial distribution of aftershocks (Aki 1979). More extensive seismic and other geophysical observations would be needed to clarify the heterogeneous properties of fault zones of impending large earthquakes, in order to use this information for earthquake prediction purposes.

It has thus been demonstrated that the existence of fault asperities or barriers, which are represented here by various types of heterogeneous distributions of strengths over a fault plane, could well explain the long-term spatial and temporal variations of seismic activity and slow fault slips before major earthquakes. It is to be emphasized, however, that actual observations should have a greater variety than discussed here. Careful investigations into much more observed data are needed before more generalizations can be made.

\section{Conclusions}

We have investigated a possible physical model to explain the observed spatial and temporal variations of seismic activity prior to large earthquakes. The model we have worked out here is for three-dimensional heterogeneous faults with various types of non-uniform frictional strength distributions, and subjected to a time-dependent shear stress. The physical parameters that play a dominant role on quasi-static and dynamic rupture processes in this model are the form of distributions of static and sliding frictional strengths and their spatial variations on the fault, and the rate of increase of the tectonic stress. The main conclusions we have obtained from the present numerical modelling are:

(1) One of the typical seismic sequences observed before major earthquakes, composed of precursory swarms, quiescence, foreshocks and a main shock event, could appear on extremely heterogeneous faults including a number of small- to moderate-size asperities with medium to high strengths, which are represented by a widely separated bi-modal strength distribution.

(2) Some versions of the above typical patterns have been observed, which sometimes lack precursory swarms or foreshock activity or both. These cases may be explained by slightly different distributions of the strengths. The time interval between these precursory events seems to depend mainly on the mode of distribution of strengths, and partly on their spatial variations.

(3) A large-scale main event rupturing the entire fault region could occur without any precursory changes of seismicity patterns, if the fault strengths are relatively homogeneous. Gradually increasing foreshock activity could also take place, without precursory swarms and quiescence, over a long period before a main shock event, if the fault has moderately to heavily non-uniform strengths on a small scale.

(4) A doughnut pattern of seismicity could appear on heterogeneous faults which include a large-size asperity with medium to higher average strengths in its central region. In this 
case, high activity or clustering and a long period of quiescence appear alternately in the surrounding and central regions.

(5) Two large shocks could take place successively within a short time interval in adjacent unruptured regions at one time, and a main large event rupturing the entire fault region could occur at another time. This could be the case if the fault has a barrier region with high strengths, dividing the entire fault into two sections where small-size asperities are almost equally distributed.

\section{Acknowledgments}

We wish to thank Professor Hitoshi Mizutani for providing us preprints and drafts before publication, and Professor Tokuji Utsu for generous permission to reproduce a figure from his paper. We have benefited from conversations with Professors Frank Evison and Hiroo Kanamori. Helpful comments and reviews on the original manuscript given by Professor Evison and anonymous reviewers are sincerely appreciated.

The numerical computations involved were made at the Data Processing Centre, Kyoto University and the Earthquake Prediction Information Centre, Earthquake Research Institute, University of Tokyo.

\section{References}

Aki, K., 1979. Characterization of barriers on an earthquake fault, J. geophys. Res., 84, 6140-6148.

Ando, M., 1975. Source mechanisms and tectonic significance of historical earthquakes along the Nankai trough, Japan, Tectonophys., 27, 119-140.

Byerlee, J., 1978. Friction of rocks, Pageoph, 116, 615-626.

Das, S. \& Aki, K., 1977. A numerical study of two-dimensional spontaneous rupture propagation, Geophys. J. R. astr. Soc., 50,643-668.

Das, S. \& Scholz, C. H., 1981. Theory of time-dependent rupture in the earth, J. geophys. Res., 86, $6039-6051$.

Dieterich, J. H., 1972. Time-dependent friction in rocks, J. geophys. Res., 77, 3690-3697.

Dieterich, J. H., 1979. Modelling of rock friction, 1. Experimental results and constitutive equations; 2. Simulation of preseismic slip, J geophys. Res., 84, 2161-2175.

Evison, F. F., 1977a. Fluctuation of seismicity before major earthquakes, Nature, 266, 710-712.

Evison, F. F., 1977b. Precursory seismic sequences in New Zealand, N.Z. J. Geol. Geophys., 20, 129141.

Evison, F. F., 1977c. The precursory earthquake swarm, Phys. Earth planet. Int., 15, 19-23.

Fedotov, S. A., 1965. Regularities of the distribution of strong earthquakes in Kamchatka, the Kurile Islands and northeastern Japan, Trans. Acad. Sci., USSR, Inst. Phys. Earth, 36, 66-93.

Fujii, Y., 1978. Phases and characteristics of seismic crustal movement with special references to Japanese earthquakes, Bull. geogr. Surv. Inst., Tokyo, 23, 7-81.

Inouye, W., 1965. On the seismicity in the epicentral region and its neighbourhood before the Niigata earthquake, $Q$. $J$ seism., Tokyo, 29, 31-36 (in Japanese).

Ishida, M. \& Kanamori, H., 1978. The foreshock activity of the 1971 San Fernando earthquake, California, Bull. seism. Soc. Am., 68, 1265-1279.

Ishida, M. \& Kanamori, H., 1980. Temporal variation of seismicity and spectrum of small earthquakes preceding the 1952 Kern County, California, earthquake, Bull. seism. Soc. Am., 70, 508-527.

Jones, L. M. \& Molnar, P., 1979. Some characteristics of foreshocks and their possible relationship to earthquake prediction and premonitory slip on faults, J. geophys. Res., 84, 3596-3608.

Kanamori, H., 1981. The nature of seismicity patterns before large earthquakes, in Earthquake Prediction - an International Review, pp. 1-19, Maurice Ewing Series 4, American Geophysical Union.

Kanamori, H. \& Cipar, J. J., 1974. Focal process of the great Chilean earthquake, May 22, 1960, Phys. Earth planet. Int., 9, 128-136.

Katsumata, M. \& Yoshida, A., 1980. Changes in seismicity and development of focal region, Pap. Met. Geophys., Tokyo, 31, 15-32.

Kelleher, J. \& Savino, J., 1975. Distribution of seismicity before large strike slip and thrust type earthquakes, J. geophys. Res., 80, 260-271. 
Kelleher, J., Sykes, L. \& Oliver, J., 1973. Possible criteria for predicting earthquake locations and their application to major plate boundaries of the Pacific and the Caribbean, J. geophys. Res., 78, 2547-2585.

Lay, T. \& Kanamori, H., 1981. An asperity model of large earthquake sequences, in Earthquake Prediction - an International Review, pp. 579-592, Maurice Ewing Series 4, American Geophysical Union.

McCann, W., Nishenko, R. S. P., Sykes, L. R.\& Kraus, J., 1979. Seismic gaps and plate tectonics: seismic potential for major boundaries, Pageoph, 117, 1082-1147.

Mikumo, T., 1981. A possible rupture process of slow earthquakes on a frictional fault, Geophys. J. $R$. astr. Soc., 65, 129-153.

Mikumo, T. \& Miyatake, T., 1978. Dynamical rupture process on a three-dimensional fault with nonuniform frictions, and near-field seismic waves, Geophys. J. R. astr. Soc., 54, 417-438.

Mikumo, T. \& Miyatake, T., 1979. Earthquake sequences on a frictional fault model with non-uniform strengths and relaxation times, Geophys. J. R. astr. Soc., 59, 497-522.

Miyatake, T., 1980. Numerical simulations of earthquake source process by a three-dimensional crack model. Part I. Rupture process, J. Phys. Earth, 28, 565-598.

Miyatake, T., 1982. Great earthquake sequence along the Nankai trough - a hypothesis, Bull. Earthq. Res. Inst. Tokyo Univ., 57, 105-109 (in Japanese).

Mogi, K., 1963a. The fracture of a semi-infinite body caused by an inner stress origin and its relation to the earthquake phenomena (second paper), Bull. Earthq. Res. Inst. Tokyo Univ., 41,595-614.

Mogi, K., 1963b. Some discussions on aftershocks, foreshocks, and earthquake swarms - the fracture of a semi-infinite body caused by an inner stress origin and its relation to the earthquake swarm (third paper), Bull. Earthq. Res. Inst. Tokyo Univ., 41, 615-658.

Mogi, K., 1968a. Some features of recent seismic activity in and near Japan, (1), Bull. Earthq. Res. Inst. Tokyo Univ., 46, 1225-1236.

Mogi, K., 1968b. Sequential occurrence of recent great earthquakes, J. Phys. Earth, 16, 30--36.

Mogi, K., 1968c. Migration of seismic activity, Bull. Earthq. Res. Inst. Tokyo Univ., 46, 53-74.

Mogi, K., 1969. Some features of recent seismic activity in and near Japan, (2) Activity before and after great earthquakes, Bull. Earthq. Res. Inst. Tokyo Univ., 47, 395-417.

Mogi, K., 1977. Seismic activity and earthquake prediction, Proc. Symp. Earthq. Pred. Res. Japan, pp. 203-214 (in Japanese).

Ohtake, M., 1976. Search for precursors of the 1974 Izu-Hanto-Oki earthquake, Japan, Pageoph, 114, 1083-1093.

Ohtake, M., Matsumoto, T. \& Latham, G. V., 1977. Seismicity gap near Oaxaca, southern Mexico as a probable precursor to a large earthquake, Pageoph, 115, 375-385.

Sacks, I. S., Suyehiro, S., Linde, A. T. \& Snoke, J. A., 1978. Slow earthquakes and stress redistribution, Nature, 275, $599-602$.

Scholz, C. H. \& Engelder, J. T., 1976. The role of asperity indentation and ploughing in rock friction - I. Asperity creep and stick-slip, Int. J. Rock Mech. Min. Sci. Geomech. Abstr., 113, 149-154.

Sekiya, H., 1977. Anomalous seismic activity and earthquake prediction, Suppl. J. Phys. Earth, 25, 8593.

Spetzler, H., Mizutani, H. \& Rummel, F., 1982. A model for time-dependent rock failure, preprint submitted to High Pressure Geoscience Research, German Science Foundation.

Sykes, L. R. 1971. Aftershock zone of great earthquakes, seismicity gaps, and earthquake prediction for Alaska and Aleutians, J. geophys. Res., 76, 8021-8041.

Sykes, L. R., Kisslinger, J. B., House, L., Davis, J. N. \& Jacob, K. H., 1980. Alaska-Aleutian arc, $1784-$ 1980, Science, 210, 1343-1345.

Utsu, T., 1974. Space-time pattern of large earthquakes occurring off the coast of the Japanese islands, J. Phys. Earth, 22, 325-342.

Utsu, T., 1977. Possibility of a great earthquake in the Tokai district, central Japan, Suppl. J. Phys. Earth, $25,219-230$.

Utsu, T., 1980. Problems associated with earthquake prediction - General remarks and some topics, Proc. Symp. Earthq. Pred. Res., Japan, pp. 202-203.

Yamaguchi, Y. \& Nishimatsu, U., 1967. Introduction to Rock Mechanics, Tokyo University Press (in Japanese).

Yamashina, K. \& Inouye, Y., 1979. A doughnut-shaped pattern of seismic activity preceding the Shimane earthquake of 1978 , Nature, 278, 48-50.

Yamashita, T., 1980. Causes of slow earthquakes and multiple earthquakes, J. Phys. Earth, 28, $169-190$. 